\title{
EIGENVALUE BOUNDS FOR THE POLY-HARMONIC OPERATORS
}

\author{
SELMA YILDIRIM YOLCU AND TÜRKAY YOLCU
}

\begin{abstract}
We study some estimates for the sums of eigenvalues of the Dirichlet poly-harmonic operator $(-\Delta)^{l}$ restricted to a bounded domain $\Omega \subset \mathbb{R}^{d}$ with $d \geq 2, l \geq 1$. Our approach yields estimates sharper than the estimates recently obtained by Q.-M. Cheng, X. Qi and G. Wei (Pacific J. Math. 262 (2013) 35-47) and G. Wei and L. Zeng (Estimates for eigenvalues of poly-harmonic operators, preprint). Another central object of study in this paper is to establish some certain estimates for the sums of powers of the eigenvalues of the poly-harmonic operator $\left.(-\Delta)^{l}\right|_{\Omega}$.
\end{abstract}

\section{Introduction}

In this article, we study estimates pertaining to the eigenvalues $\left\{\Lambda_{j}^{(l)}\right\}_{j=1}^{\infty}$ of the poly-harmonic operator restricted to $\Omega$ defined by the eigenvalue problem:

$$
\begin{aligned}
(-\Delta)^{l} w_{j}^{(l)} & =\Lambda_{j}^{(l)} w_{j}^{(l)} \quad \text { in } \Omega \\
w_{j}^{(l)} & =\frac{\partial w_{j}^{(l)}}{\partial \nu}=\cdots=\frac{\partial^{l-1} w_{j}^{(l)}}{\partial \nu^{l-1}}=0 \quad \text { on } \partial \Omega,
\end{aligned}
$$

where $\Omega$ is a bounded connected domain in $\mathbb{R}^{d}$ with smooth boundary, $d \geq 2$, $l \geq 1$, and $\nu$ denotes the outward unit normal to the boundary $\partial \Omega$. Here, the 2 th order operator $(-\Delta)^{l}$ denotes the poly-harmonic operator (or the poly-Laplacian operator) on $\mathbb{R}^{d}$ where $-\Delta$ is the Laplace operator. The poly-harmonic operator can be regarded as iterations of the Laplace operator

Received July 19, 2014; received in final form November 1, 2014.

2010 Mathematics Subject Classification. Primary 35P15. Secondary 35P20.

(C) 2015 University of Illinois 
defined inductively by [10]

$$
(-\Delta)^{l} \varphi=(-\Delta)\left((-\Delta)^{l-1} \varphi\right) \text { for } l=1,2,3, \ldots
$$

By induction on $l \geq 1$, it is shown that

$$
(-\Delta)^{l} \varphi=\sum_{l_{1}+l_{2}+\cdots+l_{d}=l} \frac{(-1)^{l} l !}{l_{1} ! l_{2} ! \cdots l_{d} !} \frac{\partial^{2 l} \varphi}{\partial x_{1}^{2 l_{1}} \partial x_{2}^{2 l_{2}} \cdots \partial x_{d}^{2 l_{d}}}
$$

The eigenvalues $\left\{\Lambda_{j}^{(l)}\right\}_{j=1}^{\infty}$ (including multiplicities) of (1), invariant under translation or rotation of the domain $\Omega$, satisfy [7], [26]

$$
0<\Lambda_{1}^{(l)} \leq \Lambda_{2}^{(l)} \leq \cdots \leq \Lambda_{k}^{(l)} \leq \cdots \rightarrow \infty
$$

When $l=1$, the operator is just the Dirichlet Laplacian, which has been extensively investigated by many authors. For example, P. Li and S.-T. Yau [18] and F. A. Berezin [5] obtained equivalent lower bounds for the sum of eigenvalues of the Dirichlet Laplacian. Later, A. Melas [20] provided an improvement to their result. More recently, the result of Melas is sharpened in [30].

When $l=2$, we recover the bi-harmonic operator (or the bi-Laplacian operator) and the boundary conditions are usually called clamped or Kirchhoff conditions [14]. In addition, in the study of small transverse vibrations of an elastic plate, the bi-harmonic operator describes the resonant frequencies [24]. The bi-harmonic operator is also the subject of many works, for instance $[1],[2],[3],[4],[12],[17],[22]$. For a comprehensive history of the clamped plate problem, see for example Henrot's book [14, Chapter 11]. Agmon [1] and Pleijel [22] established the Weyl asymptotics for the eigenvalues of the biharmonic operator. Later, Levine and Protter extended the Berezin-Li-Yau inequality for arbitrary order $l \geq 1$, for the eigenvalues of the poly-harmonic operator in [17], which is also sharp in the sense of Weyl's asymptotics. They proved that

$$
\sum_{j=1}^{k} \Lambda_{j}^{(l)} \geq(4 \pi)^{l} \frac{d}{d+2 l}\left(\frac{\Gamma\left(1+\frac{d}{2}\right)}{|\Omega|}\right)^{\frac{2 l}{d}} k^{1+\frac{2 l}{d}},
$$

where $|\Omega|$ represents the volume of $\Omega$ and $\Gamma(x)$ denotes the Gamma function $\Gamma(x)=\int_{0}^{\infty} t^{x-1} e^{-t} d t$ for $x>0$.

Even though the case $l>2$ is much less studied, it has recently received more attention. We refer the reader to papers [6], [7], [8], [9], [10], [15], [16], [17], [21], [23], [26]. For instance, look at [8], [16], [21] for Lieb-Thirring type inequalities, [15] for universal bounds for eigenvalues and [10] for some boundary value problems.

One of the recent remarkable results extending (4) is obtained by Q.-M. Cheng, X. Qi and G. Wei in [7]. In particular, they showed that the eigenvalues 
of the poly-harmonic operator explicitly satisfy

$$
\begin{aligned}
\sum_{j=1}^{k} \Lambda_{j}^{(l)} \geq & (4 \pi)^{l} \frac{d}{d+2 l}\left(\frac{\Gamma\left(1+\frac{d}{2}\right)}{|\Omega|}\right)^{\frac{2 l}{d}} k^{1+\frac{2 l}{d}} \\
& +\frac{l(4 \pi)^{l-1}}{24(d+2 l)} \frac{|\Omega|^{1-\frac{2(l-1)}{d}} \Gamma\left(1+\frac{d}{2}\right)^{\frac{2(l-1)}{d}}}{\mathcal{I}(\Omega)} k^{1+\frac{2(l-1)}{d}} \\
& +\frac{d}{d+2 l} \sum_{p=2}^{l}(4 \pi)^{l-p} \mathcal{K}_{p} \frac{|\Omega|^{p-\frac{2(l-p)}{d}} \Gamma\left(1+\frac{d}{2}\right)^{\frac{2(l-p)}{d}}}{\mathcal{I}(\Omega)^{p}} k^{1+\frac{2(l-p)}{d}}
\end{aligned}
$$

where

$$
\mathcal{K}_{p}=\frac{l+1-p}{(24)^{p} d \cdots(d+2 p-2)},
$$

and $\mathcal{I}(\Omega)$, the moment of inertia, is defined by

$$
\mathcal{I}(\Omega)=\min _{y \in \mathbb{R}^{d}} \int_{\Omega}|z-y|^{2} d z .
$$

By a translation of the origin and a rotation of axes if necessary, in the sequel, we assume that the origin is the center of mass of $\Omega$ and that

$$
\mathcal{I}(\Omega)=\int_{\Omega}|z|^{2} d z .
$$

Shortly after, in contrast to (5), G. Wei and L. Zeng give a more precise sharper estimate established in [26]:

$$
\begin{aligned}
\sum_{j=1}^{k} \Lambda_{j}^{(l)} \geq & \frac{d(4 \pi)^{l}}{d+2 l}\left(\frac{\Gamma\left(1+\frac{d}{2}\right)}{|\Omega|}\right)^{\frac{2 l}{d}} k^{1+\frac{2 l}{d}} \\
& +\frac{l(4 \pi)^{l-1}}{24(d+2 l)} \frac{|\Omega|^{1-\frac{2(l-1)}{d}} \Gamma\left(1+\frac{d}{2}\right)^{\frac{2(l-1)}{d}}}{\mathcal{I}(\Omega)} k^{1+\frac{2(l-1)}{d}} \\
& +\frac{l(d+2(l-1))^{2}(4 \pi)^{l-2}}{2304 d(d+2 l)^{2}} \frac{|\Omega|^{2-\frac{2(l-2)}{d}} \Gamma\left(1+\frac{d}{2}\right)^{\frac{2(l-2)}{d}}}{\mathcal{I}(\Omega)^{2}} k^{1+\frac{2(l-2)}{d}}
\end{aligned}
$$

The focus of this paper is to find analogues for the poly-harmonic operator of some familiar inequalities of a general nature that apply to the eigenvalues of the Dirichlet Laplacian and bi-harmonic operators on a bounded open domain $\Omega \subset \mathbb{R}^{d}$. To this end, we first improve earlier bounds of Q.-M. Cheng, X. Qi, G. Wei in [7] and G. Wei and L. Zeng in [26]. Comparable estimates have been obtained for Stokes operators and Dirichlet Laplacian in [30], for the fractional Laplacian operators in [28], [29], [32] and for the bi-harmonic operator in [31]. Precisely, we shall establish the following main result: 
TheOREM 1. For $l \geq 1, d \geq 2, k \geq 1$ the eigenvalues $\left\{\Lambda_{j}^{(l)}\right\}_{j=1}^{\infty}$ of the polyharmonic operator (1) defined on $\Omega$ satisfy

$$
\begin{aligned}
\sum_{j=1}^{k} \Lambda_{j}^{(l)} \geq & \frac{d(4 \pi)^{l}}{d+2 l}\left(\frac{\Gamma\left(1+\frac{d}{2}\right)}{|\Omega|}\right)^{\frac{2 l}{d}} k^{1+\frac{2 l}{d}} \\
& +\frac{l(4 \pi)^{l-1}}{24(d+2 l)} \frac{|\Omega|^{1-\frac{2(l-1)}{d}} \Gamma\left(1+\frac{d}{2}\right)^{\frac{2(l-1)}{d}}}{\mathcal{I}(\Omega)} k^{1+\frac{2(l-1)}{d}} \\
& +\frac{l(4 \pi)^{l-\frac{3}{2}}}{72(d+2 l)} \frac{|\Omega|^{\frac{3}{2}-\frac{2 l-3}{d}} \Gamma\left(1+\frac{d}{2}\right)^{\frac{2 l-3}{d}}}{\mathcal{I}(\Omega)^{\frac{3}{2}}} k^{1+\frac{2 l-3}{d}}
\end{aligned}
$$

Note that when we substitute $l=1$ and $l=2$ into (8), we recover the lower bounds in the case of the Dirichlet Laplacian [30] and the bi-harmonic operator [31], respectively. Also, when $l=1$, the first two terms on the right of (5), (7) and (8) yield the improvement in [20].

Let $\mathcal{N}_{l}(z)$ denote the counting function which gives the number of eigenvalues $\Lambda_{j}^{(l)}$ less than or equal to $z$. Namely,

$$
\mathcal{N}_{l}(z)=\sup _{\Lambda_{j}^{(l)} \leq z}\{j\}=\sum_{j: \Lambda_{j}^{(l)} \leq z} 1 .
$$

For $t>0$, the partition function $\mathcal{Z}_{l}(t)$ is defined as

$$
\mathcal{Z}_{l}(t):=\sum_{j=1}^{\infty} e^{-\Lambda_{j}^{(l)} t}
$$

In terms of the counting function $\mathcal{N}_{l}(z), \mathcal{Z}_{l}(t)$ can also be written as

$$
\mathcal{Z}_{l}(t)=\int_{0}^{\infty} e^{-z t} d \mathcal{N}_{l}(z)
$$

Next, we establish upper bounds for the sums of positive as well as negative powers of eigenvalues, the counting function and the partition function. Precisely, we shall prove the following estimates:

THEOREM 2. For $0<\eta \leq 1, l \geq 1$ and $d \geq 2$, the sums of positive powers of eigenvalues of the poly-harmonic operator on $\Omega$ satisfy

$$
\sum_{j=1}^{k}\left[\Lambda_{j}^{(l)}\right]^{\eta} \geq(4 \pi)^{l \eta} \frac{d}{d+2 l \eta}\left(\frac{\Gamma\left(1+\frac{d}{2}\right)}{|\Omega|}\right)^{\frac{2 l \eta}{d}} k^{1+\frac{2 l \eta}{d}} .
$$

Corollary 3. Let $l \geq 1, d \geq 2$. The counting function $\mathcal{N}_{l}$ for the polyharmonic operator on $\Omega$ has the following upper bound for $z \geq 0$ :

$$
\mathcal{N}_{l}(z) \leq \frac{(d+2 l)^{\frac{d}{2 l}}}{d^{\frac{d}{2 l}}} \frac{1}{(4 \pi)^{\frac{d}{2}}} \frac{|\Omega|}{\Gamma\left(1+\frac{d}{2}\right)} z^{\frac{d}{2 l}} .
$$


COROllary 4. Let $l \geq 1, d \geq 2$. The partition function $\mathcal{Z}_{l}$ for the polyharmonic operator on $\Omega$ has the following upper bound for $t \geq 0$ :

$$
\mathcal{Z}_{l}(t) \leq \frac{(d+2 l)^{\frac{d}{2 l}}}{d^{\frac{d}{2 l}}} \frac{|\Omega| \Gamma\left(1+\frac{d}{2 l}\right)}{(4 \pi)^{\frac{d}{2}} \Gamma\left(1+\frac{d}{2}\right)} t^{-\frac{d}{2 l}} .
$$

TheOrem 5. For $0<\rho<\frac{d}{2 l}, l \geq 1$ and $d \geq 2$, the sums of negative powers of eigenvalues of the poly-harmonic operator on $\Omega$ satisfy

$$
\sum_{j=1}^{k}\left[\Lambda_{j}^{(l)}\right]^{-\rho} \leq(4 \pi)^{-l \rho} \frac{d}{d-2 l \rho}\left(\frac{|\Omega|}{\Gamma\left(1+\frac{d}{2}\right)}\right)^{\frac{2 l \rho}{d}} k^{1-\frac{2 l \rho}{d}} .
$$

The article is organized as follows: In Section 2, we collect some facts on the eigenvalues and eigenfunctions of the poly-harmonic operator that will play a central role in proving Theorem 1. Section 3 presents some intermediate steps for the proof of Theorem 1. Section 4 reports on the derivation of improved Berezin-Li-Yau type inequality on a general domain $\Omega$ as well as certain inequalities involving sums of positive and negative powers of the eigenvalues of the poly-harmonic operator. We also present and prove a Kac type inequality by an application of the Laplace transform.

\section{Preliminaries}

In this section, we provide a summary of the relevant theory previously developed in [27]. This lays the foundation for establishing the estimates in (8) and (12). Throughout this article, $B_{R}(x):=\left\{y \in \mathbb{R}^{d}:|x-y| \leq R\right\}$ denotes the ball of radius $R$ centered at $x$ in $\mathbb{R}^{d}$ and $\omega_{d}$ denotes the volume of $d$ dimensional unit ball $B_{1}(x)$ in $\mathbb{R}^{d}$ given by

$$
\omega_{d}=\frac{\pi^{\frac{d}{2}}}{\Gamma\left(1+\frac{d}{2}\right)} .
$$

Thus, the surface area of the unit ball in $\mathbb{R}^{d}$ is $d \omega_{d}$.

Let us now review some well-known properties of the eigenfunctions of the poly-harmonic operator. First, note that the Dirichlet Laplacian operator on $\Omega \subset \mathbb{R}^{d}$ can be conveniently defined by using the Fourier transform $\mathcal{F}$ on the dense subspace of test functions $C_{c}^{\infty}\left(\mathbb{R}^{d}\right)$ as

$$
\left.(-\Delta)\right|_{\Omega} \varphi=\mathcal{F}^{-1}\left[|\boldsymbol{\xi}|^{2} \mathcal{F}\left[\varphi \chi_{\Omega}\right]\right]
$$

which together with (2) yields a convenient definition for the poly-harmonic operator on $\Omega \subset \mathbb{R}^{d}$ as follows:

$$
\left.(-\Delta)^{l}\right|_{\Omega} \varphi=\mathcal{F}^{-1}\left[|\xi|^{2 l} \mathcal{F}\left[\varphi \chi_{\Omega}\right]\right],
$$


where $\chi_{\Omega}$ is the characteristic function on $\Omega$ and the Fourier transform and its inverse are defined as

$$
\mathcal{F}[\varphi](\xi)=\frac{1}{(2 \pi)^{\frac{d}{2}}} \int_{\mathbb{R}^{d}} e^{-i \xi \cdot z} \varphi(z) d z, \quad \mathcal{F}^{-1}[\varphi](\eta)=\frac{1}{(2 \pi)^{\frac{d}{2}}} \int_{\mathbb{R}^{d}} e^{i \xi \cdot \eta} \varphi(\xi) d \xi .
$$

The set of Fourier transforms $\left\{\mathcal{F}\left[w_{j}^{(l)}(\xi)\right]\right\}_{j=1}^{\infty}$ of $\left\{w_{j}^{(l)}\right\}_{j=1}^{\infty}$ forms an orthonormal set in $L^{2}\left(\mathbb{R}^{d}\right)$ since the set of eigenfunctions $\left\{w_{j}^{(l)}\right\}_{j=1}^{\infty}$ is an orthonormal set in $L^{2}(\Omega)$. This result is due to Plancherel's theorem. To ease the notation, henceforth, we set

$$
\mathcal{W}_{k}(\xi):=\sum_{j=1}^{k}\left|\mathcal{F}\left[w_{j}^{(l)}(\xi)\right]\right|^{2}=\frac{1}{(2 \pi)^{d}} \sum_{j=1}^{k}\left|\int_{\Omega} e^{-i z \cdot \xi} w_{j}^{(l)}(z) d z\right|^{2} \geq 0 .
$$

Here, the integral is taken over $\Omega$ instead of $\mathbb{R}^{d}$ because the support of $w_{j}^{(l)}$ is $\Omega$. Interchanging the sum and integral and using $\left\|\mathcal{F}\left[w_{j}^{(l)}(\xi)\right]\right\|_{2}=1$, we obtain

$$
\int_{\mathbb{R}^{d}} \mathcal{W}_{k}(\xi) d \xi=k
$$

In addition, an application of the Bessel's inequality yields the following upper bound for $\mathcal{W}_{k}$ :

$$
\mathcal{W}_{k}(\xi) \leq \frac{1}{(2 \pi)^{d}} \int_{\Omega}\left|e^{-i z \cdot \xi}\right|^{2} d z=\frac{|\Omega|}{(2 \pi)^{d}}
$$

Observe that

$$
\begin{aligned}
\Lambda_{j}^{(l)} & =\left\langle w_{j}^{(l)}, \Lambda_{j}^{(l)} w_{j}^{(l)}\right\rangle \\
& =\left\langle w_{j}^{(l)},(-\Delta)^{l} w_{j}^{(l)}\right\rangle \\
& =\left\langle w_{j}^{(l)}, \mathcal{F}^{-1}\left[|\xi|^{2 l} \mathcal{F}\left[w_{j}^{(l)}\right]\right]\right\rangle \\
& =\int_{\mathbb{R}^{d}}|\xi|^{2 l}\left|\mathcal{F}\left[w_{j}^{(l)}(\xi)\right]\right|^{2} d \xi .
\end{aligned}
$$

Summing over $j$ from 1 to $k$ we see that $\mathcal{W}_{k}$ defined by (15) also satisfies

$$
\int_{\mathbb{R}^{d}}|\xi|^{2 l} \mathcal{W}_{k}(\xi) d \xi=\sum_{j=1}^{k} \Lambda_{j}^{(l)}
$$

Now, we find an estimate for $\left|\nabla \mathcal{W}_{k}\right|$. Note that

$$
\sum_{j=1}^{k}\left|\nabla \mathcal{F}\left[w_{j}^{(l)}(\xi)\right]\right|^{2} \leq \frac{1}{(2 \pi)^{d}} \int_{\Omega}\left|i z e^{-i z \cdot \xi}\right|^{2} d z=\frac{\mathcal{I}(\Omega)}{(2 \pi)^{d}} .
$$


Using Hölder's inequality, we obtain that for every $\xi$,

$$
\left|\nabla \mathcal{W}_{k}(\xi)\right| \leq 2\left(\sum_{j=1}^{k}\left|\mathcal{F}\left[w_{j}^{(l)}(\xi)\right]\right|^{2}\right)^{1 / 2}\left(\sum_{j=1}^{k}\left|\nabla \mathcal{F}\left[w_{j}^{(l)}(\xi)\right]\right|^{2}\right)^{1 / 2}
$$

Invoking (17) and (20), we end up with

$$
\left|\nabla \mathcal{W}_{k}(\xi)\right| \leq \beta:=2(2 \pi)^{-d} \sqrt{|\Omega| \mathcal{I}(\Omega)} .
$$

Now assume that $R$ is such that $|\Omega|=\omega_{d} R^{d}$. That is, $B_{R}(0)$ is the symmetric rearrangement of $\Omega$. Note that

$$
\mathcal{I}(\Omega) \geq \int_{B_{R}(0)}|z|^{2} d z=\frac{d \omega_{d}}{d+2} R^{d+2}=\frac{d}{d+2} \omega_{d}^{-\frac{2}{d}}|\Omega|^{\frac{d+2}{d}},
$$

leading to

$$
\beta \geq \frac{|\Omega|^{\frac{d+1}{d}}}{(2 \pi)^{d} \omega_{d}^{\frac{1}{d}}}
$$

In addition, supposing that $\mathcal{W}_{k}^{*}(\xi)$ denotes the decreasing radial rearrangement of $\mathcal{W}_{k}(\xi)$, by approximating $\mathcal{W}_{k}$, we may infer that there exists a real valued absolutely continuous function $\varphi_{k}:[0, \infty) \rightarrow\left[0,(2 \pi)^{-d}|\Omega|\right]$ such that

$$
\mathcal{W}_{k}^{*}(\xi)=\varphi_{k}(|\xi|) \text {. }
$$

Define the distribution function $\gamma_{k}$ by

$$
\gamma_{k}(s):=\left|\left\{\mathcal{W}_{k}(\xi)>s\right\}\right|=\left|\left\{\mathcal{W}_{k}^{*}(\xi)>s\right\}\right| .
$$

Then, $\gamma_{k}\left(\varphi_{k}(t)\right)=\omega_{d} t^{d}$. In fact,

$$
\gamma_{k}\left(\varphi_{k}(t)\right)=\left|\left\{\mathcal{W}_{k}^{*}(\xi)>\varphi_{k}(t)\right\}\right|=|\{\xi:|\xi|<t\}|=\left|B_{t}(0)\right|=\omega_{d} t^{d} .
$$

Utilizing Federer's coarea formula with the aid of (17), we have

$$
\begin{aligned}
\gamma_{k}(s) & =\int_{s}^{\infty} \int_{\left\{\mathcal{W}_{k}^{-1}(t)\right\}} \frac{1}{\left|\nabla \mathcal{W}_{k}\right|} d \mathcal{H}^{d-1} d t \\
& =\int_{s}^{(2 \pi)^{-d}|\Omega|} \int_{\left\{\mathcal{W}_{k}=t\right\}} \frac{1}{\left|\nabla \mathcal{W}_{k}\right|} d \mathcal{H}^{d-1} d t,
\end{aligned}
$$

where $\mathcal{H}^{d-1}$ is the $(d-1)$ dimensional Hausdorff measure. The isoperimetric inequality,

$$
\mathcal{H}^{d-1}(\partial \Omega) \geq d \omega_{d}^{\frac{1}{d}}|\bar{\Omega}|^{\frac{d-1}{d}}, \quad \Omega \subset \mathbb{R}^{d},
$$

together with $\varphi_{k}^{\prime}(t) \leq 0, t \geq 0$, leads to the following observation

$$
\begin{aligned}
d \omega_{d} t^{d-1} & =\gamma_{k}^{\prime}\left(\varphi_{k}(t)\right) \varphi_{k}^{\prime}(t) \\
& =-\varphi_{k}^{\prime}(t) \int_{\left\{\mathcal{W}_{k}=\varphi_{k}(t)\right\}} \frac{1}{\left|\nabla \mathcal{W}_{k}\right|} d \mathcal{H}^{d-1}
\end{aligned}
$$




$$
\begin{aligned}
\operatorname{by}(21) & \geq-\frac{1}{\beta} \mathcal{H}^{d-1}\left(\left\{\mathcal{W}_{k}=\varphi_{k}(t)\right\}\right) \varphi_{k}^{\prime}(t) \\
\operatorname{by}(25) & \geq-\frac{d \omega_{d}^{\frac{1}{d}}}{\beta} \gamma_{k}\left(\varphi_{k}(t)\right)^{\frac{d-1}{d}} \varphi_{k}^{\prime}(t) \\
& =-\frac{d \omega_{d} t^{d-1}}{\beta} \varphi_{k}^{\prime}(t) .
\end{aligned}
$$

This inequality combined with $\varphi_{k}^{\prime} \leq 0$ simply means $0 \leq-\varphi_{k}^{\prime}(t) \leq \beta$.

\section{Key lemmas for the proof of Theorem 1}

Our method of proof is similar in spirit to those explored in [20], [27], [30], [31] with significant differences. Among the many results obtained is the following surprising sharper inequality which will be the main ingredient in the proof of the refined lower bound in (8) while Q.-M. Cheng, X. Qi and G. Wei in [7] and G. Wei and L. Zheng [26] suitably use Taylor series expansion in weaker inequalities.

LEMMA 6. For integers $d \geq 2, l \geq 1$ and real numbers $0<\varepsilon \leq 1, p>0$, $q>0$, we have the following inequality

$$
\begin{aligned}
& d p^{d+2 l}-(d+2 l) p^{d} q^{2 l}+2 l q^{d+2 l} \\
& \quad-2 l \varepsilon q^{d+2 l-2}(p-q)^{2}-4 l \varepsilon p q^{d+2 l-3}(p-q)^{2} \geq 0 .
\end{aligned}
$$

To make the exposition self-contained, a short proof is presented below.

Proof of Lemma 6. Define

$$
\Sigma_{d, l}(\alpha):=d \alpha^{d+2 l}-(d+2 l) \alpha^{d}+2 l-2 l(1+2 \alpha)(\alpha-1)^{2} .
$$

Simply using double induction on the integers $d \geq 2$ and $p \geq 1$, we obtain that

$$
\Sigma_{d, l}(\alpha)=(\alpha-1)^{2}\left[\sum_{k=2}^{d-2} 2 l(k+1) \alpha^{k}+\sum_{k=2}^{2 l+1} d(k-1) \alpha^{d+2 l-k}\right] \geq 0
$$

for any $\alpha>0$. Since the right side of (27) is non-negative, setting $\alpha=p / q$ and multiplying the resulting expression by $q^{d+2 l}$ we obtain

$$
d p^{d+2 l}-(d+2 l) p^{d} q^{2 l}+2 l q^{d+2 l}-2 l q^{d+2 l-2}(p-q)^{2}-4 l p q^{d+2 l-3}(p-q)^{2} \geq 0 .
$$

Since $0<\varepsilon \leq 1$, we conclude $(26)$, as required.

Having Lemma 6 at hand, we are in a position to show the following useful estimate that can be regarded as a precursor to the proof of Theorem 1 . 
Lemma 7. Assume that (16)-(21) hold and $d \geq 2, l \geq 1$. For any real number $\varepsilon \in(0,1]$ and each integer $k \geq 1$

$$
\begin{aligned}
\sum_{j=1}^{k} \Lambda_{j}^{(l)} \geq & \frac{d}{d+2 l} \omega_{d}^{-\frac{2 l}{d}} \varphi_{k}(0)^{-\frac{2 l}{d}} k^{1+\frac{2 l}{d}} \\
& +\frac{l \varepsilon}{6(d+2 l)} \beta^{-2} \omega_{d}^{-\frac{2 l-2}{d}} \varphi_{k}(0)^{2-\frac{2 l-2}{d}} k^{1+\frac{2 l-2}{d}} \\
& +\frac{l \varepsilon}{9(d+2 l)} \beta^{-3} \omega_{d}^{-\frac{2 l-3}{d}} \varphi_{k}(0)^{3-\frac{2 l-3}{d}} k^{1+\frac{2 l-3}{d}} .
\end{aligned}
$$

Proof of Lemma 7. Consider the decreasing, absolutely continuous function $\varphi_{k}:[0, \infty) \rightarrow[0, \infty)$ defined by (24). Recall that $0 \leq-\varphi_{k}^{\prime}(t) \leq \beta$ for $t \geq 0$ where $\beta>0$ is given by (23). By (15), we know that $\varphi_{k}(0)>0$. Set

$$
\mathcal{G}_{k}(p):=\frac{1}{\varphi_{k}(0)} \varphi_{k}\left(\frac{\varphi_{k}(0)}{\beta} p\right) .
$$

Notice that $\mathcal{G}_{k}$ is positive, $\mathcal{G}_{k}(0)=1$ and $0 \leq-\mathcal{G}_{k}^{\prime}(p) \leq 1$. For ease of notation, we also define $g_{k}(p):=-\mathcal{G}_{k}^{\prime}(p)$ for $t \geq 0$. Hence, $0 \leq g_{k}(p) \leq 1$ for $t \geq 0$ and

$$
\int_{0}^{\infty} g_{k}(p) d p=\mathcal{G}_{k}(0)=1 \text {. }
$$

Let us define

$$
\lambda_{k}=\int_{0}^{\infty} p^{d-1} \mathcal{G}_{k}(p) d p \quad \text { and } \quad \zeta_{k}=\int_{0}^{\infty} p^{d+2 l-1} \mathcal{G}_{k}(p) d p .
$$

With the aid of (16), we obtain

$$
k=\int_{\mathbb{R}^{d}} \mathcal{W}_{k}(\xi) d \xi=\int_{\mathbb{R}^{d}} \mathcal{W}_{k}^{*}(\xi) d \xi=d \omega_{d} \int_{0}^{\infty} p^{d-1} \varphi_{k}(p) d p .
$$

In addition, (19) together with the fact that the map $\xi \mapsto|\xi|^{2 l}$ is radial and increasing, we get

$$
\begin{aligned}
\sum_{j=1}^{k} \Lambda_{j}^{(l)} & =\int_{\mathbb{R}^{d}}|\xi|^{2 l} \mathcal{W}_{k}(\xi) d \xi \geq \int_{\mathbb{R}^{d}}|\xi|^{2 l} \mathcal{W}_{k}^{*}(\xi) d \xi \\
& =d \omega_{d} \int_{0}^{\infty} p^{d+2 l-1} \varphi_{k}(p) d p .
\end{aligned}
$$

Substitution of (29) into (30) yields

$$
\begin{aligned}
\lambda_{k} & =\frac{\beta^{d}}{\varphi_{k}(0)^{d+1}} \int_{0}^{\infty} p^{d-1} \varphi_{k}(p) d p=\frac{\beta^{d} k}{d \omega_{d} \varphi_{k}(0)^{d+1}}, \\
\zeta_{k} & =\frac{\beta^{d+2 l}}{\varphi_{k}(0)^{d+2 l+1}} \int_{0}^{\infty} p^{d+2 l-1} \varphi_{k}(p) d p \leq \frac{\beta^{d+2 l} \sum_{j=1}^{k} \Lambda_{j}^{(l)}}{d \omega_{d} \varphi_{k}(0)^{d+2 l+1}} .
\end{aligned}
$$


For a finite integer $k \geq 1$ it is clear that $\zeta_{k}<+\infty$. Suppose that $p^{d+2 l} \mathcal{G}_{k}(p) \rightarrow$ $L>0$ as $p \rightarrow \infty$. Then for any $0<C<L$ we can find a finite number $R>0$ such that

$$
0<\frac{L-C}{p}<p^{d+2 l-1} \mathcal{G}_{k}(p)<\frac{L+C}{p}
$$

for any $p>R$. Therefore, using (34) we arrive at a contradiction:

$$
\infty=\int_{R}^{\infty} p^{d+2 l-1} \mathcal{G}_{k}(p) d p \leq \int_{0}^{\infty} p^{d+2 l-1} \mathcal{G}_{k}(p) d p<\infty,
$$

as $0 \leq \zeta_{k}<\infty$. Thus, $p^{d+2 l} \mathcal{G}_{k}(p) \rightarrow 0$ as $p \rightarrow \infty$. Moreover, in the same vein, it is not difficult to observe that $p^{d+2 l-1} \mathcal{G}_{k}(p) \rightarrow 0$ as $p \rightarrow \infty$, as well. Thus, applications of integration by parts simply yield

$$
\int_{0}^{\infty} p^{d} g_{k}(p) d p=\lambda_{k} d \quad \text { and } \quad \int_{0}^{\infty} p^{d+2 l} g_{k}(p) d p=\zeta_{k}(d+2 l)
$$

Notice that

$$
\left(p^{d}-1\right)\left(g_{k}(p)-\chi_{[0,1]}(p)\right) \geq 0, \quad p \in[0, \infty) .
$$

Integrating (36) from 0 to $\infty$ yields

$$
\int_{0}^{\infty} p^{d} g_{k}(p) d p \geq \frac{1}{d+1}=\phi_{d}(0)
$$

where $\phi_{d}:[0, \infty) \rightarrow(0, \infty)$ is defined by

$$
\phi_{d}(a)=\int_{a}^{a+1} p^{d} d p
$$

Since $\phi_{d}$ is continuous and non-decreasing and $\phi_{d}(a) \rightarrow \infty$ as $a \rightarrow \infty$, the Intermediate Value theorem provides us with the existence of $\varrho \geq 0$ such that

$$
\phi_{d}(\varrho)=\int_{\varrho}^{\varrho+1} p^{d} d p=\int_{0}^{\infty} p^{d} g_{k}(p) d p
$$

which, by (35), concludes that

$$
\int_{\varrho}^{\varrho+1} p^{d} d p=d \lambda_{k}
$$

Now consider the polynomial

$$
\mathcal{Q}(p)=p^{d+2 l}-\alpha_{1} p^{d}+\alpha_{2}=p^{d}\left(p^{2 l}-\alpha_{1}\right)+\alpha_{2}
$$

where

$$
\alpha_{1}=\frac{(\varrho+1)^{d+2 l}-\varrho^{d+2 l}}{(\varrho+1)^{d}-\varrho^{d}}>0, \quad \alpha_{2}=\frac{(\varrho+1)^{d+2 l}-\varrho^{d+2 l}}{(\varrho+1)^{d}-\varrho^{d}} \varrho^{d}-\varrho^{d+2 l} \geq 0
$$

are chosen so that $\mathcal{Q}(\varrho)=0$ and $\mathcal{Q}(\varrho+1)=0$ and $\mathcal{Q}$ remains negative on $(\varrho, \varrho+1)$ and positive on $[0, \infty) \backslash[\varrho, \varrho+1]$. It is immediate to observe that

$$
\mathcal{Q}(p)\left(\chi_{[\varrho, \varrho+1]}(p)-g_{k}(p)\right) \leq 0 \quad \text { on }[0, \infty) .
$$


Integration of $(38)$ on $[0, \infty)$ leads to

$$
\int_{\varrho}^{\varrho+1} p^{d+2 l} d p \leq \int_{0}^{\infty} p^{d+2 l} g_{k}(p) d p-\alpha_{1}\left(\int_{0}^{\infty} p^{d} g_{k}(p) d p-\int_{\varrho}^{\varrho+1} p^{d} d p\right),
$$

simplifying to

$$
\int_{\varrho}^{\varrho+1} p^{d+2 l} d p \leq \int_{0}^{\infty} p^{d+2 l} g_{k}(p) d p .
$$

Using (35), we infer that

$$
\int_{\varrho}^{\varrho+1} p^{d+2 l} d p \leq \zeta_{k}(d+2 l) .
$$

Observe that Jensen's inequality leads to

$$
d \lambda_{k}=\int_{\varrho}^{\varrho+1} p^{d} d p \geq\left(\int_{\varrho}^{\varrho+1} p d p\right)^{d} \geq\left(\int_{0}^{1} p d p\right)^{d}=\frac{1}{2^{d}} .
$$

Notice that (26) gives the crucial ingredient in the proof of this lemma. Indeed, integrating (26) in $p$ from $\varrho$ to $\varrho+1$ we arrive at

$$
\begin{aligned}
\int_{\varrho}^{\varrho+1} p^{d+2 l} d p \geq & \frac{d+2 l}{d} q^{2 l} \int_{\varrho}^{\varrho+1} p^{d} d p-\frac{2 l}{d} q^{d+2 l} \\
& +\frac{2 l \varepsilon}{d} q^{d+2 l-2} \int_{\varrho}^{\varrho+1}(p-q)^{2} d p \\
& +\frac{4 l \varepsilon}{d} q^{d+2 l-3} \int_{\varrho}^{\varrho+1} p(p-q)^{2} d p .
\end{aligned}
$$

Now, for any $q \geq 1 / 2$ and $\varrho \geq 0$, it is not difficult to see that

$$
\begin{gathered}
\int_{\varrho}^{\varrho+1}(p-q)^{2} d p \geq \min _{\varrho \geq 0, q \geq 1 / 2} \int_{\varrho}^{\varrho+1}(p-q)^{2} d p=\frac{1}{12} \\
\int_{\varrho}^{\varrho+1} p(p-q)^{2} d p \geq \min _{\varrho \geq 0, q \geq 1 / 2} \int_{\varrho}^{\varrho+1} p(p-q)^{2} d p=\frac{1}{36} .
\end{gathered}
$$

Since $\left(\lambda_{k} d\right)^{1 / d} \geq 1 / 2$ due to (41), setting $s=\left(\lambda_{k} d\right)^{1 / d}$ and using (37), (40), (43) and (44), we deduce that (42) simplifies to

$$
\zeta_{k} \geq \frac{1}{d+2 l}\left(\lambda_{k} d\right)^{1+\frac{2 l}{d}}+\frac{l \varepsilon}{6 d(d+2 l)}\left(\lambda_{k} d\right)^{1+\frac{2 l-2}{d}}+\frac{l \varepsilon}{9 d(d+2 l)}\left(\lambda_{k} d\right)^{1+\frac{2 l-3}{d}}
$$

which holds true for any $0<\varepsilon \leq 1$. Thus, we obtain (28) from (45) by utilizing equations in (33) together with (31) and (32). This finishes the proof of Lemma 7.

\section{Proof of main results}

This section reports the proofs of our main results. 
4.1. Proof of Theorem 1. Now, we are ready to prove Theorem 1 by using Lemmas 6 and 7 .

Proof of Theorem 1. The starting point is to minimize the right side of $(28)$ over $\varphi_{k}(0)$. To do this, let us first set $x=\varphi_{k}(0)>0$. By (17), recall that we know $0<x \leq(2 \pi)^{-d}|\Omega|$. Also, set

$$
\begin{aligned}
\delta_{1} & =\frac{d k^{1+\frac{2 l}{d}}}{(d+2 l) \omega_{d}^{\frac{2 l}{d}}}, \quad \delta_{2}^{\varepsilon}=\frac{l \varepsilon k^{1+\frac{2 l-2}{d}}}{6(d+2 l) \beta^{2} \omega_{d}^{\frac{2 l-2}{d}}}, \\
\delta_{3}^{\varepsilon} & =\frac{l \varepsilon k^{1+\frac{2 l-3}{d}}}{9(d+2 l) \beta^{3} \omega_{d}^{\frac{2 l-3}{d}}},
\end{aligned}
$$

and define

$$
\mathcal{S}(x)=\delta_{1} x^{-\frac{2 l}{d}}+\delta_{2}^{\varepsilon} x^{2-\frac{2 l-2}{d}}+\delta_{3}^{\varepsilon} x^{3-\frac{2 l-3}{d}} .
$$

Since $d \geq 2$ and $l \geq 1$, we have

$$
2-\frac{2 l-2}{d}<3-\frac{2 l-3}{d}
$$

Our aim is to show that $\mathcal{S}$ decreases on $\left(0,(2 \pi)^{-d}|\Omega|\right]$ even if $\varepsilon=1$. To this end, we need to analyze the following three cases:

Case $I: d<2 l / 3-1$. It is not difficult to see that all powers of $x$ in (47) are negative and so $\mathcal{S}$ is decreasing. Namely, $\mathcal{S}(x) \geq \mathcal{S}\left((2 \pi)^{-d}|\Omega|\right)$. Therefore, taking $\varepsilon=1$ and substituting $\beta=2(2 \pi)^{-d} \sqrt{|\Omega| \mathcal{I}(\Omega)}$ into (47), we obtain the right side of (8).

Case $I I: 2 l / 3-1 \leq d<l-1$. This means that

$$
2-\frac{2 l-2}{d}<0 \quad \text { and } \quad 3-\frac{2 l-3}{d} \geq 0
$$

We define $\mathcal{S}_{1}:\left(0,(2 \pi)^{-d}|\Omega|\right] \rightarrow(0, \infty)$ by

$$
\mathcal{S}_{1}(x)=\delta_{2}^{\varepsilon} x^{2-\frac{2 l-2}{d}}+\delta_{3}^{\varepsilon} x^{3-\frac{2 l-3}{d}} .
$$

After differentiating $\mathcal{S}_{1}$, we see that $\mathcal{S}_{1}(x)$ is decreasing when

$$
0<x \leq\left(\frac{(2 l-2 d-2) \delta_{2}^{\varepsilon}}{(3 d-2 l+3) \delta_{3}^{\varepsilon}}\right)^{\frac{d}{d+1}}=\left(\frac{3(l-d-1) \beta \omega_{d}^{\frac{1}{d}} k^{\frac{1}{d}}}{3 d-2 l+3}\right)^{\frac{d}{d+1}}
$$

Using $-d \leq 1-2 l / 3$ we obtain that

$$
\frac{1}{2 \pi}<\frac{3(l-d-1)}{3 d-2 l+3}
$$

Using $\beta \omega_{d}^{\frac{1}{d}} \geq(2 \pi)^{-d}|\Omega|^{\frac{d+1}{d}}$ together with (49), we obtain that

$$
\frac{|\Omega|}{(2 \pi)^{d}} \leq\left(\frac{3(l-d-1) \beta \omega_{d}^{\frac{1}{d}} k^{\frac{1}{d}}}{3 d-2 l+3}\right)^{\frac{d}{d+1}}
$$


for any $k \geq 1$ and $0<\varepsilon \leq 1$. Thus, $\mathcal{S}_{1}$ is decreasing on $\left(0,(2 \pi)^{-d}|\Omega|\right]$ for any value of $\varepsilon \in(0,1]$. Thus, $\mathcal{S}(x)=\mathcal{S}_{1}(x)+\delta_{1} x^{-\frac{2 l}{d}}$ is also decreasing on $\left(0,(2 \pi)^{-d}|\Omega|\right]$. As a result, we may take $\varepsilon=1$ and, as in the previous case, we obtain the desired inequality in (8) from $\mathcal{S}(x) \geq \mathcal{S}\left((2 \pi)^{-d}|\Omega|\right)$.

Case III: $d \geq l-1$. In this case,

$$
2-\frac{2 l-2}{d}>0 \text { and } 3-\frac{2 l-3}{d}>0
$$

Here, the clincher is to separately use the monotonicity of two functions $\mathcal{S}_{2}, \mathcal{S}_{3}:\left(0,(2 \pi)^{-d}|\Omega|\right] \rightarrow(0, \infty)$ defined by

$$
\mathcal{S}_{2}(x)=\frac{\delta_{1}}{2} x^{-\frac{2 l}{d}}+\delta_{2}^{\varepsilon} x^{2-\frac{2 l-2}{d}}, \quad \mathcal{S}_{3}(x)=\frac{\delta_{1}}{2} x^{-\frac{2 l}{d}}+\delta_{3}^{\varepsilon} x^{3-\frac{2 l-3}{d}} .
$$

Differentiating $\mathcal{S}_{2}$ and $\mathcal{S}_{3}$, we observe that $\mathcal{S}_{2}(x)$ is decreasing when $0<x \leq$ $\left(\frac{l \delta_{1}^{\varepsilon}}{(2 d-2 l+2) \delta_{2}^{\varepsilon}}\right)^{\frac{d}{2 d+2}}$ while $\mathcal{S}_{3}(x)$ is decreasing when $0<x \leq\left(\frac{l \delta_{1}^{\varepsilon}}{(3 d-2 l+3) \delta_{3}^{\varepsilon}}\right)^{\frac{d}{3 d+3}}$. Hence, we particularly obtain that $x \mapsto\left(\mathcal{S}_{2}+\mathcal{S}_{3}\right)(x)=\mathcal{S}(x)$ is decreasing on $\left(0,(2 \pi)^{-d}|\Omega|\right]$ when we have

$$
\frac{|\Omega|}{(2 \pi)^{d}} \leq \min \left\{\left(\frac{6 d \beta^{2} k^{\frac{2}{d}}}{\varepsilon(2 d-2 l+2) \omega_{d}^{\frac{2}{d}}}\right)^{\frac{d}{2 d+2}},\left(\frac{9 d \beta^{3} k^{\frac{3}{d}}}{\varepsilon(3 d-2 l+3) \omega_{d}^{\frac{3}{d}}}\right)^{\frac{d}{3 d+3}}\right\}
$$

for any $k \geq 1$. In other words, in view of the inequalities $2 d-2 l+2 \leq 2 d$, $3 d-2 l+3 \leq 3 d+1, \beta \geq(2 \pi)^{-d} \omega_{d}^{-\frac{1}{d}}|\Omega|^{\frac{d+1}{d}}$ and definition of $\omega_{d}$ given in (13) we may take $\varphi_{k}(0)=(2 \pi)^{-d}|\Omega|$ when we have

$$
\varepsilon \leq \min _{d \geq 2}\left\{\mathcal{T}_{1}(d), \mathcal{T}_{2}(d)\right\}
$$

where

$$
\mathcal{T}_{1}(d)=12\left[\Gamma\left(1+\frac{d}{2}\right)\right]^{\frac{4}{d}}, \quad \mathcal{T}_{2}(d)=\frac{72 d}{3 d+1}\left[\Gamma\left(1+\frac{d}{2}\right)\right]^{\frac{6}{d}} .
$$

Note that $\mathcal{T}_{1}(d)$ and $\mathcal{T}_{2}(d)$, running over integers greater than or equal to 2 , assume their minimum at $d=2$ with values $\mathcal{T}_{1}(2)=12$ and $\mathcal{T}_{2}(2)=144 / 7$, respectively (see Figure 1). As a result of these observations, we can replace $\varphi_{k}(0)$ with $|\Omega|(2 \pi)^{-d}$ in $(28)$ when we set

$$
\varepsilon=\min \left\{1, \mathcal{T}_{1}(d), \mathcal{T}_{2}(d)\right\}=1 .
$$

In conclusion, $\mathcal{S}(x) \geq \mathcal{S}\left((2 \pi)^{-d}|\Omega|\right)$ and substitution of $\beta=2(2 \pi)^{-d} \sqrt{|\Omega| \mathcal{I}(\Omega)}$ given in (23) together with $\varphi_{k}(0)=|\Omega|(2 \pi)^{-d}$ turn (28) into (8).

REMARK 1. In [26], it is shown that (7) is sharper than (5). Now, we elucidate why (8) is sharper than (7). Clearly, the power of $k$ in the last term in (8) is bigger than the power of $k$ in the last term in (7). However, due to 

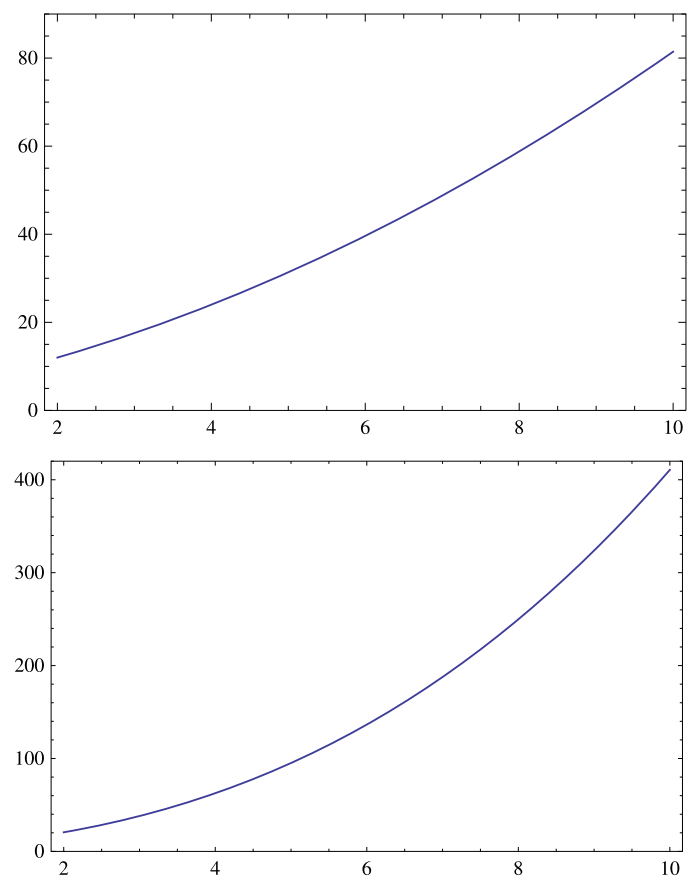

FIGURE 1. Graphs of $\mathcal{T}_{1}(d)$ and $\mathcal{T}_{2}(d)$ respectively.

the uncertainty of $|\Omega|$ and $I[\Omega]$, we can not directly compare the coefficients in $(7)$ and $(8)$. Note that (22) leads to

$$
\sqrt{\frac{\mathcal{I}(\Omega)}{|\Omega|^{\frac{d+2}{d}}}} \geq \sqrt{\frac{d}{\pi(d+2)}}\left[\Gamma\left(1+\frac{d}{2}\right)\right]^{\frac{1}{d}} .
$$

Simple calculations together with (52) show that (8) is sharper than (7) for

$$
k \geq \frac{(d+2(l-1))^{2 d}(d+2)^{d / 2}}{2^{6 d} d^{3 d / 2}(d+2 l)^{d}\left[\Gamma\left(1+\frac{d}{2}\right)\right]^{2}}:=M(d, l) .
$$

One can compute that

$$
\max _{2 \leq d \leq 10^{7}, 1 \leq l \leq 46} M(d, l)=M(2,46)=0.989705 .
$$

This observation tells us that, for any integer $k \geq 1$, (8) provides us with a better lower bound than the one in (7) for at least $2 \leq d \leq 10^{7}$ and $1 \leq l \leq 46$. While it must be admitted that the inequality in (8) is arguably not the sharpest in theory, one can make it stronger possibly for higher dimensions by exploiting an analogous technique employed here. 
Moreover, it is interesting to ask if there is a term involving $k^{1+\frac{2 l-1}{d}}$, which surprisingly does not exist in recent works.

4.2. Proof of Theorem 2. The following proof is inspired from the proof of Berezin-Li-Yau inequality in [13], [18], [29], [30]. An analogous proof is also exploited in [25] by means of Bathtub principle [19].

Proof of Theorem 2. Recall that $\mathcal{W}_{k}$ satisfies (16)-(19). Using the fact that $\left|\mathcal{F}\left[w_{j}^{(l)}(\xi)\right]\right|^{2} d \xi$ is a probability measure on $\mathbb{R}^{d}$ and that $z \mapsto z^{\eta}$ is concave for $z>0$ and $0<\eta \leq 1$, we apply Jensen's inequality to derive that

$$
\sum_{j=1}^{k}\left[\Lambda_{j}^{(l)}\right]^{\eta}=\sum_{j=1}^{k}\left(\int_{\mathbb{R}^{d}}|\xi|^{2 l}\left|\mathcal{F}\left[w_{j}^{(l)}(\xi)\right]\right|^{2} d \xi\right)^{\eta} \geq \int_{\mathbb{R}^{d}}|\xi|^{2 l \eta} \mathcal{W}_{k}(\xi) d \xi
$$

Setting

$$
\mathcal{A}_{k}(\xi)=\frac{|\Omega|}{(2 \pi)^{d}} \chi_{B_{a_{k}}(0)}(\xi)
$$

where

$$
a_{k}=\left(\frac{(d+2 l \eta)(2 \pi)^{d}\left(\sum_{j=1}^{k}\left[\Lambda_{j}^{(l)}\right]^{\eta}\right)}{d \omega_{d}|\Omega|}\right)^{\frac{1}{d+2 l \eta}}
$$

we have

$$
\int_{\mathbb{R}^{d}}|\xi|^{2 l \eta} \mathcal{A}_{k}(\xi) d \xi=\sum_{j=1}^{k}\left[\Lambda_{j}^{(l)}\right]^{\eta}
$$

Now observe that

$$
\left(|\xi|^{2 l \eta}-a_{k}^{2 l \eta}\right)\left(\mathcal{W}_{k}(\xi)-\mathcal{A}_{k}(\xi)\right) \geq 0
$$

Integrating (57) on $\mathbb{R}^{d}$ and using (56), we conclude that

$$
a_{k}^{2 l \eta} \int_{\mathbb{R}^{d}}\left(\mathcal{W}_{k}(\xi)-\mathcal{A}_{k}(\xi)\right) d \xi \leq \int_{\mathbb{R}^{d}}|\xi|^{2 l \eta}\left(\mathcal{W}_{k}(\xi)-\mathcal{A}_{k}(\xi)\right) d \xi \leq 0
$$

which simply yields

$$
\int_{\mathbb{R}^{d}} \mathcal{W}_{k}(\xi) d \xi \leq \int_{\mathbb{R}^{d}} \mathcal{A}_{k}(\xi) d \xi
$$

Thus, by (16), we obtain

$$
k \leq \int_{\mathbb{R}^{d}} \mathcal{A}_{k}(\xi) d \xi=\frac{|\Omega|}{(2 \pi)^{d}} d \omega_{d}\left(\frac{a_{k}^{d}}{d}\right) .
$$

Substituting $\omega_{d}$ given by (13) and $a_{k}$ given by (55) into (59) and simplifying the terms, we therefore deduce the inequality in (9), as required.

It is worth noting that setting $\eta=1$ in (9) recovers the estimate in (4). 
4.3. Proof of Theorem 5. Even though the following proof is done in a similar vein, we sketch the details for the sake of completeness as it does not directly follow from the previous one.

Proof of Theorem 5. Assume the properties (16)-(19). Since $\left|\mathcal{F}\left[w_{j}^{(l)}(\xi)\right]\right|^{2} d \xi$ is a probability measure on $\mathbb{R}^{d}$ and $t \mapsto t^{-\rho}$ is convex for $t>0$ and $\rho>0$, employing Jensen's inequality and (15), we obtain that

$$
\sum_{j=1}^{k}\left[\Lambda_{j}^{(l)}\right]^{-\rho}=\sum_{j=1}^{k}\left(\int_{\mathbb{R}^{d}}|\xi|^{2 l}\left|\mathcal{F}\left[w_{j}^{(l)}(\xi)\right]\right|^{2} d \xi\right)^{-\rho} \leq \int_{\mathbb{R}^{d}} \frac{\mathcal{W}_{k}(\xi)}{|\xi|^{2 l \rho}} d \xi
$$

Define

$$
\mathcal{B}_{k}(\xi)=\frac{|\Omega|}{(2 \pi)^{d}} \chi_{B_{b_{k}}(0)}(\xi), \quad b_{k}=\left(\frac{(d-2 l \rho)(2 \pi)^{d}\left(\sum_{j=1}^{k}\left[\Lambda_{j}^{(l)}\right]^{-\rho}\right)}{d \omega_{d}|\Omega|}\right)^{\frac{1}{d-2 l \rho}}
$$

so that

$$
\int_{\mathbb{R}^{d}} \frac{\mathcal{B}_{k}(\xi)}{|\xi|^{2 l \rho}} d \xi=\sum_{j=1}^{k}\left[\Lambda_{j}^{(l)}\right]^{-\rho}
$$

Notice that

$$
\left(\frac{1}{|\xi|^{2 l \rho}}-\frac{1}{b_{k}^{2 l \rho}}\right)\left(\mathcal{W}_{k}(\xi)-\mathcal{B}_{k}(\xi)\right) \leq 0
$$

Integrating (62) on $\mathbb{R}^{d}$ and using (61), we arrive at

$$
\frac{1}{b_{k}^{2 l \rho}} \int_{\mathbb{R}^{d}}\left(\mathcal{W}_{k}(\xi)-\mathcal{B}_{k}(\xi)\right) d \xi \geq \int_{\mathbb{R}^{d}} \frac{\left(\mathcal{W}_{k}(\xi)-\mathcal{B}_{k}(\xi)\right)}{|\xi|^{2 l \rho}} d \xi \geq 0
$$

from which it follows that

$$
\int_{\mathbb{R}^{d}} \mathcal{W}_{k}(\xi) d \xi \geq \int_{\mathbb{R}^{d}} \mathcal{B}_{k}(\xi) d \xi .
$$

Thus, by (16), we obtain

$$
k \geq \int_{\mathbb{R}^{d}} \mathcal{B}_{k}(\xi) d \xi=\frac{|\Omega|}{(2 \pi)^{d}} d \omega_{d}\left(\frac{b_{k}^{d}}{d}\right) .
$$

Replacing $\omega_{d}$ and $b_{k}$ with their actual values in (64) and rearranging the terms, we infer the demanded inequality in (12).

Now, we shall sketch the proof of corollaries. 


\subsection{Proofs of Corollaries 3-4.}

Proofs of Corollaries $3-4$. To see (10), we take $z \in\left[\Lambda_{k}^{(l)}, \Lambda_{k+1}^{(l)}\right)$ so that $\mathcal{N}_{l}(z)=k$. Employing (9) with $\eta=1$ leads to

$$
z \geq \frac{1}{k}\left(k \Lambda_{k}^{(l)}\right) \geq \frac{1}{k} \sum_{j=1}^{k} \Lambda_{j}^{(l)} \geq(4 \pi)^{l} \frac{d}{d+2 l}\left(\frac{\Gamma\left(1+\frac{d}{2}\right)}{|\Omega|}\right)^{\frac{2 l}{d}} k^{\frac{2 l}{d}}
$$

and so

$$
\mathcal{N}_{l}(z) \leq \frac{(d+2 l)^{\frac{d}{2 l}}}{d^{\frac{d}{2 l}}} \frac{1}{(4 \pi)^{\frac{d}{2}}} \frac{|\Omega|}{\Gamma\left(1+\frac{d}{2}\right)} z^{\frac{d}{2 l}}
$$

as desired.

Next, we suitably utilize the Laplace transform to obtain a Kac type inequality as in [11]. To this end, let us first recall the definition of the Laplace transform:

$$
\mathcal{L}[\psi(z)](t)=\int_{0}^{\infty} e^{-z t} \psi(z) d z
$$

It is not difficult to see that

$$
\mathcal{L}\left[\mathcal{N}_{l}(z)\right](t)=\frac{\mathcal{Z}_{l}(t)}{t} \quad \text { and } \quad \mathcal{L}\left[z^{\frac{d}{2 l}}\right](t)=\frac{\Gamma\left(1+\frac{d}{2 l}\right)}{t^{1+\frac{d}{2 l}}} .
$$

Thus, taking the Laplace transform of both sides of (10) leads to (11).

Acknowledgments. The author would like to thank the anonymous referee for his/her comments on a preliminary version of this manuscript.

\section{REFERENCES}

[1] S. Agmon, On kernels, eigenvalues and eigenfunctions of operators related to elliptic problems, Comm. Pure Appl. Math. 18 (1965), 627-663. MR 0198287

[2] M. Ashbaugh and R. Benguria, On Rayleigh's conjecture for the clamped plate and its generalization to three dimensions, Duke Math. J. 78 (1995), 1-17. MR 1328749

[3] M. Ashbaugh, R. Benguria and R. Laugesen, Inequalities for the first eigenvalues of the clamped plate and buckling problems, General inequalities 7 (C. Bandle, W. N. Everitt, L. Losonczi and W. Walter, eds.), International Series of Numerical Mathematics, vol. 123, Birkhäuser, Basel, 1997, pp. 95-110. MR 1457272

[4] M. Ashbaugh and R. Laugesen, Fundamental tones and buckling loads of clamped plates, Ann. Sci. Éc. Norm. Supér. (4) 23 (1996), 383-402. MR 1433428

[5] F. A. Berezin, Covariant and contravariant symbols of operators, Izv. Akad. Nauk SSSR Ser. Mat. 36 (1972), 1134-1167. MR 0350504

[6] D. Buoso and P. D. Lamberti, Eigenvalues of poly-harmonic operators on variable domains, ESAIM Control Optim. Calc. Var. 19 (2013), no. 4, 1225-1235. MR 3182687

[7] Q.-M. Cheng, X. Qi and G. Wei, A lower bound for eigenvalues of the poly-Laplacian with arbitrary order, Pacific J. Math. 262 (2013), no. 1, 35-47. MR 3069054

[8] C. Förster and J. Ostensson, Lieb-Thirring inequalities for higher order differential operators, Math. Nachr. 281 (2008), no. 2, 199-213. MR 2387360

[9] H. C. Grunau, The Dirichlet problem for some semilinear elliptic differential equations of arbitrary order, Analysis 11 (1991), 83-90. MR 1113070 
[10] F. Gazzola, H.-C. Grunau and G. Sweers, Polyharmonic boundary value problems. Positivity preserving and nonlinear higher order elliptic equations in bounded domains, Lecture Notes in Mathematics, vol. 1991, Springer, Berlin, 2010. MR 2667016

[11] E. M. Harrell II and L. Hermi, On Riesz means of eigenvalues, Comm. Partial Differential Equations 36 (2011), 1521-1543. MR 2825601

[12] E. M. Harrell II and P. L. Michel, Commutator bounds for eigenvalues of some differential operators, Lecture Notes in Pure and Applied Mathematics, Dekker, New York, 1993. MR 1300432

[13] E. M. Harrell II and S. Yıldırım Yolcu, Eigenvalue inequalities for Klein-Gordon operators, J. Funct. Anal. 256 (2009), 3977-3995. MR 2521917

[14] A. Henrot, Extremum problems for eigenvalues of elliptic operators, Frontiers in Mathematics, Birkhäuser, Basel, 2006. MR 2251558

[15] J. Jost, X. Li-Jost, Q. Wang and C. Xia, Universal bounds for eigenvalues of the poly-harmonic operators, Trans. Amer. Math. Soc. 363 (2011), no. 4, 1821-1854. MR 2746667

[16] A. Laptev and T. Weidl, Recent results on Lieb-Thirring inequalities, Journées équations aux dérivées partielles, vol. XX, 14, Univ. Nantes, Nantes, 2000. MR 1775696

[17] H. A. Levine and M. H. Protter, Unrestricted lower bounds for eigenvalues for classes of elliptic equations and systems of equations with applications to problems in elasticity, Math. Methods Appl. Sci. 7 (1985), no. 2, 210-222. MR 0797333

[18] P. Li and S.-T. Yau, On the Schrödinger equation and the eigenvalue problem, Comm. Math. Phys. 88 (1983), 309-318. MR 0701919

[19] E. H. Lieb and M. Loss, Analysis, 2nd ed., Graduate Studies in Mathematics, vol. 14, Amer. Math. Soc., Providence, 2001. MR 1817225

[20] A. D. Melas, A lower bound for sums of eigenvalues of the Laplacian, Proc. Amer. Math. Soc. 131 (2002), no. 2, 631-636. MR 1933356

[21] Y. Netrusov and T. Weidl, On Lieb-Thirring inequalities for higher order operators with critical and subcritical powers, Comm. Math. Phys. 182 (1996), 355-370. MR 1447297

[22] A. Pleijel, On the eigenvalues and eigenfunctions of elastic plates, Comm. Pure Appl. Math. 3 (1950), 1-10. MR 0037459

[23] P. Pucci and J. Serrin, Remark on the first eigenspace for polyharmonic operators, Atti Semin. Mat. Fis. Univ. Modena Reggio Emilia 36 (1988), 107-117. MR 0956782

[24] S. S. Rao, Vibration of continuous systems, Wiley, Hoboken, NJ, 2007; available at http://www.wiley.com/WileyCDA/WileyTitle/productCd-0471771716.html.

[25] V. Vougalter, Sharp semiclassical bounds for the moments of eigenvalues for some Schroedinger type operators with unbounded potentials, Math. Model. Nat. Phenom. 8 (2013), no. 1, 237-245. MR 3022994

[26] G. Wei and L. Zeng, Estimates for eigenvalues of poly-harmonic operators, preprint, 2011; available at arXiv:1111.3115.

[27] S. Yıldırım Yolcu, An improvement to a Berezin-Li-Yau type inequality, Proc. Amer. Math. Soc. 138 (2010), no. 11, 4059-4066. MR 2679626

[28] S. Yıldirım Yolcu and T. Yolcu, Estimates for the sums of eigenvalues of the fractional Laplacian on a bounded domain, Commun. Contemp. Math. 15 (2013), no. 3, 1250048. MR 3063552

[29] S. Ylldirım Yolcu and T. Yolcu, Sharper estimates on the eigenvalues of Dirichlet fractional Laplacian, Discrete Contin. Dyn. Syst. 35 (2015), 2209-2225. MR 3294247

[30] S. Yıldırım Yolcu and T. Yolcu, Multidimensional lower bounds for the eigenvalues of Stokes and Dirichlet Laplacian operators, J. Math. Phys. 53 (2012), no. 4, 043508. MR 2953152 
[31] S. Yildırım Yolcu and T. Yolcu, Estimates on the eigenvalues of the clamped plate problem on domains in Euclidean spaces, J. Math. Phys. 54 (2013), no. 4, 43515. MR 3088817

[32] T. Yolcu, Refined bounds for the eigenvalues of the Klein-Gordon operator, Proc. Amer. Math. Soc. 141 (2013), no. 12, 4305-4315. MR 3105872

Selma Yildirim Yolcu, Department of Mathematics, Bradley University, PeoRIA, IL 61625, USA

E-mail address: syildirimyolcu@fsmail.bradley.edu

Türkay Yolcu, Department of Mathematics, Bradley University, Peoria, IL 61625, USA

E-mail address: tyolcu@fsmail.bradley.edu 